\title{
A Multiphase Process Approach to the Analysis of the Reliability and Safety in Maritime Transport Systems
}

\author{
D. Chybowska \\ Maritime University of Szczecin, Szczecin, Poland \\ S. Guze \\ Gdynia Maritime University, Gdynia, Poland
}

\begin{abstract}
The main aim of the paper is to develop an original approach to the analysis of the reliability and safety of maritime transport systems and the processes. It is done with accordance to multiphase process approach to reliability and safety analysis. To achieve this goal, the basic knowledge of the reliability theory of technical systems was discussed. The multiphase systems and damage trees were also characterized. The next steps of the developed solution were discussed using the s / v Ramdas sea disaster. Finally, a summary of the obtained results is presented.
\end{abstract}

\section{INTRODUCTION}

The global societies and economies depend on the safe and reliable transport of cargo, goods, and people. Modern transport systems are becoming more and more complex. Apart from their structural complexity, technical systems may be characterized by the complexity of the operation process. The system can operate differently at different stages of its operation. It also happens that the operating process of a technical system consists of a series of separate time intervals in which various tasks (processes) are performed. According to the literature ([6], [9], [19]), such these systems are defined as complex multiplephased mission systems (MPMS). This type of systems is considered in many practical applications such as aerospace, nuclear power, airborne weapon systems, and distributed computing systems. The system mission involves multiple, consecutive, and non-overlapping phases of operation ([8], [9], [16], [20], [23] - [24]). During each phase, the system must accomplish a specified task and be subject to different stresses and environmental conditions and reliability requirements ([8], [9], [20], [23] - [24]). This type of systems is characterized by the following properties [16]:

- task carried out in the phase may differ from the tasks in the remaining phases,

- performance and reliability requirements may vary between phases,

- during some phases, the system may be subjected to powerful environmental influences, which may significantly increase the intensity of damage,

- the structure of the system may change as a function of time, depending on the functional and reliability requirements formulated for the phase that is currently being performed,

- the correct execution of tasks in each phase can bring other effects for the system than those obtained in other phases.

There are two approaches to considering the operation process in the analysis and modelling of MPMS. One, the so-called synthetic model, which cover the entire system's operation process. The second approach is models in which individual phases are considered separately. However, it must be remembered that building a synthetic model is not 
easy. On the other hand, when every phase is considered separately, there is a need to consider the phases' relationship. In modelling the reliability of systems with dependencies, several results can be adapted to MPMS (e.g. [2], [4] - [6]).

According to data, the most effective mode to move the large quantities of cargo is maritime transport. However, this mode of transport is also one of the more vulnerable to changes in hydrometeorological conditions and human errors. The combination of several unfavourable factors can lead to a sea disaster. It can result in material losses, environmental pollution and, worst of all, human losses. Due to these consequences, it is so important to conduct research into methods to improve maritime safety [11], [13], [17]. One of the ways is to analyze maritime accidents and draw appropriate conclusions from them.

Thus, the article's main goal is to develop an original approach to the analysis of the reliability and safety of maritime transport systems and the processes taking place in them. The result of the assumed goal is a stepwise method of dealing with the accident data collection, which leads to the estimation of the probability of a marine accident.

The content of the article is divided as follows. Section 2 contains basic notations and well-known results for reliability modelling of technical systems and failure trees. In contrast, Section 3 contains the main result of the work presented in the example of a selected sea disaster. The work ends with conclusions and tips for further work.

\section{BASICS NOTATIONS AND METHODS}

The well-known information about the reliability of technical systems and the analysis of fault trees presented in this section constitute the basis for implementing the above-mentioned objective.

\subsection{Basics on systems reliability}

When analyzing the reliability of technical systems, we can choose between two basic approaches: twostate, multi-state. The first assumes that the system is working or not. On the other hand, the multi-state approach presents a fuller and closer to the analysed system's real picture. In this case, concerning the system and its elements, we assume that it is an ordered set of their states, where 0 is considered the worst, and $\mathrm{z}$ - the best, in terms of reliability. It can also be assumed that the two-state approach is a special case of a multi-state, assuming that $z=1$. Then the previously indicated set will contain only two states 0 and 1 . In theory, it presented, inter alia, in the works [4], [14] it is taken into account that the system and its components deteriorate during their operation unless they are repaired.

With these assumptions, vector functions of element and system reliability were defined in the literature relating to multi-state complex technical systems' reliability theory. According to them [14]:
- reliability function of components $E_{i}, \quad i=1,2, \ldots, n$ is described by the vector:

$R_{i}(t, \cdot)=\left[R_{i}(t, 0), R_{i}(t, 1), \ldots, R_{i}(t, z)\right]$

where

$R_{i}(t, u)=P\left(e_{i}(t) \geq u \mid e_{i}(0)=z\right)$

is the probability of the event that the component $E_{i}, i=1,2, \ldots, n$, at time $\mathrm{t}$, is in one of the states of the subset $\{u, u+1, \ldots, z\}$, while at time $t=0$ it was in the best state $-z$;

- system's reliability function is given as the vector:

$\mathbf{R}(t, \cdot)=[\mathbf{R}(t, 0), \mathbf{R}(t, 1), \ldots, \mathbf{R}(t, z)]$,

where

$\mathbf{R}(t, u)=P(s(t) \geq u \mid s(0)=z)$

is the probability of the event that the system at time $t$, is in one of the states of the subset while at time $t=0$ it was in the best state $-z$.

Probability that the component $E_{i}, i=1,2, \ldots, n$ is in state $u$ if at time $t=0$ it was in state $z$ is calculate as the following vector:

$p_{i}(t, \cdot)=\left[p_{i}(t, 0), p_{i}(t, 1), \ldots, p_{i}(t, z)\right]$,

where:

$p_{i}(t, u)=P\left(e_{i}(t)=u \mid e_{i}(0)=z\right)$,

for $t \in<0,+\infty), \quad u=1,2, \ldots, z$.

On the other hand, the probability that the system is in the state $\mathrm{u}$ under the condition that at time $t=0$ it was in the state $z$ is given by the vector:

$p(t, \cdot)=[p(t, 0), p(t, 1), \ldots, p(t, z)]$,

where:

$p(t, u)=P(s(t)=u \mid s(0)=z)$,

for $t \in<0,+\infty), \quad u=1,2, \ldots, z$.

The introduction of the above concepts allows defining the reliability structures of multi-state, ageing technical systems and their components and the parameters characterizing these systems. In this paper we consider only two of them, series and parallel.

\section{Definition 1}

A multi-state technical system is called a serial system if the expression

$T(u)=\min _{1 \leq i \leq n}\left\{T_{i}(u)\right\}, \quad u=1,2, \ldots, z$

describes the time $T(u)$ of its stay in a subset of reliability states $\{u, u+1, \ldots, z\}$, 
According to above definition, a system is in a reliability state subset if every component of this system is in this subset.

\section{Definition 2}

A multi-state technical system is called a parallel system if the expression

$T(u)=\max _{1 \leq i \leq n}\left\{T_{i}(u)\right\}, \quad u=1,2, \ldots, z$

describes the time $T(u)$ of its stay in a subset of reliability states.

From definition 2, it follows that the system is in a subset of reliability states $\{u, u+1, \ldots, z\}$, if at least one of its elements resides in it.

Regarding the reliability structures defined above, the coordinates of the reliability function vector are as follows [14]:

- for multi-state series system:

$$
\left.\boldsymbol{R}(t, u)=\prod_{i=1}^{n} R_{i}(t, u), \quad t \in<0,+\infty\right), \quad u=1,2, \ldots, z,
$$

- for multi-state parallel system:

$$
\left.\boldsymbol{R}(t, u) \quad 1-\prod_{i=1}^{n} F_{i}(t, u), \quad t \in<0,+\infty\right), \quad u=1,2, \ldots, z
$$

Furthermore, if we assume, that $\mathrm{u}=1$ in definitions 1,2 and in formulae (1) - (12), the reliability of the two-state technical system is described.

Extremely popular method in the reliability analysis of a technical system is reliability block diagram (RBD). One of the advantages of this method is possibility to determine the critical component form reliability point of view. This method also indirectly determines the necessity to indicate the components essential for ensuring the continuity of the system operation in the described structure. The figures 1 and 2 presents the exemplary RBD for series and parallel systems, respectively.

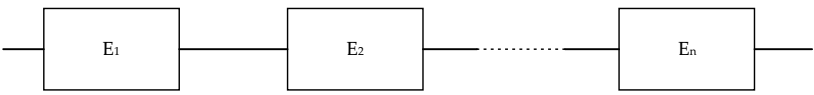

Figure 1. The exemplary series system

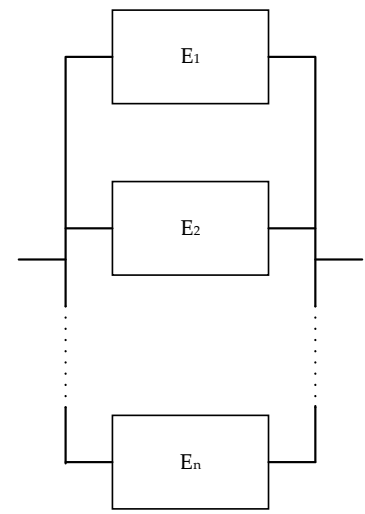

Figure 2. The exemplary parallel system
Identification of the most important components into the system can be provided with following significance measures:

- Birnbaum's measure of component importance:

$I_{C}^{B}(t)=\frac{\partial R_{S}(t)}{\partial R_{C}(t)}$

- criticality importance measure:

$I_{C}^{C}(t)=\frac{\partial R_{S}(t)}{\partial R_{C}(t)} \cdot \frac{\left(1-R_{C}(t)\right)}{\left(1-R_{S}(t)\right)}=I_{C}^{B}(t) \cdot \frac{\left(1-R_{C}(t)\right)}{\left(1-R_{S}(t)\right)}$

- reliability importance measure:

$I_{E_{i}}^{R}(t)=\frac{N S F_{E_{i}}(t)}{N S F_{S}(t)}$

where $R_{S}(t)$ is a system reliability and $R_{C}(t)$ describes component reliability, $N S F_{E_{i}}(t)$ is number of system failures caused by component $E_{i}, N S F_{S}(t)$ is a total number of system failure, $i=1,2, \ldots, n$ $t \in<0,+\infty)$.

\subsection{Fault Tree Analysis basic concepts}

The Fault Tree Analysis (FTA) is the technique which is based on the probability theory and Boolean logic [21], [22]. The first element allows estimating of the system failure probability. Also, it is possible to describe it as the function of time. On the other hand, the Boolean logic's principles reduce the fault tree structure and show the combination of events that lead to the system's failure (top event). There are some logic gates. Two most used are AND logic and OR logic gates. The figure 3 presents their symbols.

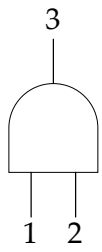

(a)

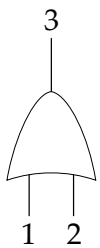

(b)
Figure 3. The exemplary logic gates in FTA: (a) AND; (b) OR.

When the failure probability is describing as a function of time, this method can estimate the function of the system's risk level. This is one of the basic characteristics, apart from the technical system's reliability, which allows rationalizing the safety of the system operation. In many publications, the risk function describes combination (product, not multiplication!) of the occurrence probability of an event and its effects ([15], [21], [22]).

To understand how calculate the top event probability I the FT, we assume that $p_{1}, p_{2}$ and are $p_{3}$ the probabilities of the events $1-3$ given in figure 3 . The event number 3 is the top one. Then the formula for occurrence probability of this event is given as follows: 
- AND gate

$p_{3}=p_{1} p_{2}$,

- OR gate:

$p_{3}=1-\left[\left(1-p_{1}\right)\left(1-p_{2}\right)\right]$

When we look at formulas 16-17, a certain observation arises. In combining reliability and risk analysis using reliability block diagrams and failure trees, the use of the serial structure determines the AND gate. For a parallel structure, use an OR gate.

\subsection{Introduction to Multiple-Phased Mission Systems}

Generally, the multiple-phased systems (MPS) are defined as systems whose operational process can be divided into several disjoint periods, called phases [25]. Considering the results given in [9], the phased mission system (PMS) is defined as a system whose relevant configuration (block diagram or fault-tree) changes during consecutive time periods (phases). In other words, it is a sequence of tasks performed to achieve the purpose of the system. A different subset of the system may need running to perform each task. During the phase operation, component or subsystem can fail at any time. If these elements of the particular phase's system are not critical, it will not affect the system performance. But considering the domino effect, if a critical component of one phase is failed in the previous one, it can lead to this phase and whole mission failure. In this case, the transition from one phase to the next in sequence is a critical event. Different features (e. g. reliability, unreliability) may be of interest during various phases, according to the specific task being performed in that phase and the user's need. Each phase is identified by phase number, time interval, system configuration, the task to be performed, a parameter of interest and maintenance policy.

\section{APPLICATION AND DISCUSSION}

Now, we introduce some new approach to disaster analysis. This method bases on the mixture of the tools introduced in Section 2 and approach to disaster process analysis defined in [12].

We show the procedure on the example of one of the thirty analyzed sea disasters, described in [1].

The first step, after collecting data from reports, is categorization into the following five phases [12]:

- phase I - latent phase $(L P h)$;

- phase II - initiating phase $(I P h)$;

- phase III - escalating phase $(E P h)$;

- phase IV - critical phase $(C P h)$;

- phase $\mathrm{V}$ - energy release $(E n P h)$.

Let us consider the disaster of s/v Ramdas on 17 July 1947 off India's coast in the Arabian Sea, several nautical miles from Bombay's port. The course of this catastrophe was as follows.
As the elementary event in $L P h$, we distinguish a problem with the lack of forecasts concerning bad hydrometeorological conditions on the planned ship's route.

Thus, the ship is in storm conditions. On the other hand, the crew tried to change the ship's course in vain. This classifies as the Iph.

Due to the above, two high waves hit the starboard. This is the single element of EPh.

To protect themselves from the waves, passengers go to the port board. As a result, the ship increases tilt in that direction. This is the critical phase. Next, the ship loses stability within 2 minutes and is sinking. As a result of this catastrophe, 669 people died. And according to [11] it is energy release phase.

This short description of the disaster helps to classify individual events into larger, thematically coherent classes of events. Particularly, we define them as follows:

- KI - Knowledge incomplete / No knowledge;

- EHC - Extreme hydrometeorological conditions;

- INM - Inefficient navigational maneuver;

- NPF - Negative physical factors;

- CSC - Change of stability characteristics;

- ST - Ship tilt;

- LS - Loss of stability;

- LL - Loss of life;

- SS - Ship sinking.

Based on above shortcuts and taking into account the results in [11] there is possibility to build the block diagram of this disaster (see figure 5).

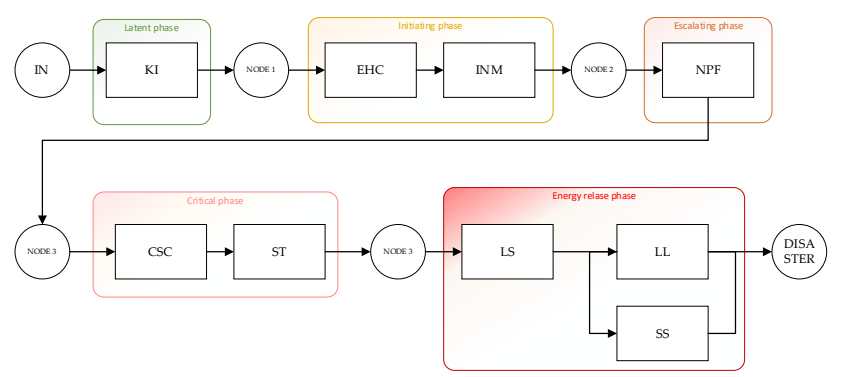

Figure 5. Block diagram of s/v Ramdas disaster

The nodes $1-4$ presented in figure 5 can be treated as barriers between stages of a modeled catastrophe.

Next step in the proposed procedure is to build a fault tree of disaster. This should be done based on a block diagram that is built from the collected data. The fault tree for s/v Ramdas disaster is in figure 6.

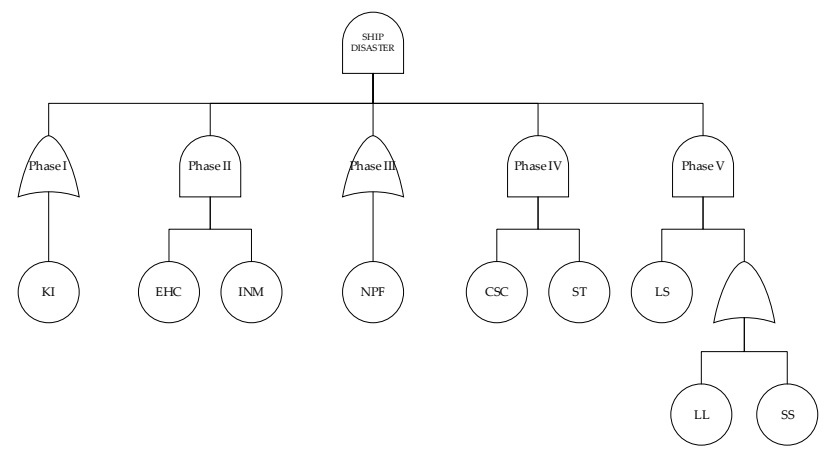

Figure 6. Fault Tree for s/v Ramdas disaster 
Considering the FT and formulae (16) - (17) the probability of the top event can be calculated. The defined classes of events make up a discrete set. Therefore, the discrete approach to the probability of events should be used. In our case, the probability of event describes following formula:

$P\left(e_{i}\right)=\frac{n_{X X X}}{N}$

where

$e_{i}$ - $i$-th event from class $\mathrm{XX}$ or $\mathrm{XXX}$ (defined above);

$n_{X X X}$ - number of all incidents belonging to class $X X$ or XXX in the total disaster population;

$N$ - total number of events in disaster population.

The value of top event's probability is equal to 0.0128

Next step is to calculate the importance, criticality, and improvement potential measures. When the discrete values are given, then the formulae (13) - (14) have to be redrawn with (18) as follows:

- discrete Birnbaum's measure of component importance:

$I_{C}^{D B}\left(e_{i}\right)=\frac{\partial P\left(e_{P h}\right)}{\partial P\left(e_{i}\right)}$,

- discrete criticality importance measure:

$I_{C}^{D C}\left(e_{i}\right)=I_{C}^{D B}\left(e_{i}\right) \cdot \frac{P\left(e_{i}\right)}{P\left(e_{P h}\right)}$,

where

$e_{i}-i$-th event in particular phase $\mathrm{Ph}$,

$e_{P h}$ - an intermediate event corresponding to the implementation of phase $\mathrm{F}$ of the disaster.

Furthermore, the expression defines the improvement potential as follows:

$I P\left(e_{i}\right)=I_{C}^{D B}\left(e_{i}\right) \cdot P\left(e_{i}\right)$,

where $I_{C}^{D B}\left(e_{i}\right)$ and $P\left(e_{i}\right)$ are given in (19), (18), respectively.

Based on formulae (18) - (21), the numerical results for $\mathrm{s} / \mathrm{v}$ Ramdas disaster are given in table 1.

Table 1. Importance of events in particular phases of the disaster.

\begin{tabular}{lccl}
\hline Event & $\begin{array}{l}\text { Birnbaum's } \\
\text { importance } \\
\text { measure }\end{array}$ & $\begin{array}{l}\text { Criticality } \\
\text { measure }\end{array}$ & $\begin{array}{l}\text { Improvement } \\
\text { potential }\end{array}$ \\
\hline $\begin{array}{l}\text { Latent phase } \\
\text { KI }\end{array}$ & 1,0000 & 1,0000 & 0,0148 \\
$\begin{array}{l}\text { Initiating phase } \\
\text { INM }\end{array}$ & 0,0544 & 1,0000 & 0,0017 \\
EHC & 0,0320 & 1,0000 & 0,0017 \\
Escalating phase & & & \\
NPF & 1,0000 & 1,0000 & 0,0444 \\
Critical phase & & & \\
CSC & 0,0496 & 1,0000 & 0,0018 \\
ST & 0,0353 & 1,0000 & 0,0018 \\
Energy release phase & & \\
LS & 0,0645 & 1,0000 & 0,0014 \\
SS & 0,0217 & 0,5289 & 0,0008 \\
LL & 0,0216 & 0,4545 & 0,0007 \\
\hline
\end{tabular}

After determining these measures, for each phase of a disaster, it is possible to determine the ranking of the importance of events, including the abovementioned definitions and the resulting impact of a given event on the occurrence of a given disaster phase. Finally, also, it is possible to calculate the impact of the entire disaster.

\section{CONCLUSION}

In the paper, the concept of tool to post-disaster analysis has been introduced. This tool has based on multiple-phase system concepts, reliability theory and fault tree analysis. It has been performed as the mixture of these elements. Thus, some well-known concepts, definitions and notations in reliability theory and fault tree analysis have been described.

Presented way of thinking allows finding information on the probability that a catastrophe would not happen. The importance measures of events in a particular phase can help improve maritime transport safety.

Further work will concern preparing a simulation tool to conduct these analyses for a specific class of sea disasters.

\section{REFERENCES}

[1] Australian Associated Press 669 Die in Ship Disaster Available online: https://trove.nla.gov.au/newspaper/article/26402001 (accessed on Feb 26, 2020).

[2] B. Babiarz, A. Blokus, "Dependency of technological lines in reliability analysis of heat production," ENERGY, vol. 211, art. no. 118593, pp. 1-17, 2020.

[3] Z.W. Birnbaum, "On the importance of different components in a multicomponent system," in Multivariate Analysis, P. R. Krishnaiah, Ed: Academic Press, 1969, vol. 11.

[4] A. Blokus, "Multistate System Reliability with Dependencies," 1st edn. Elsevier Academic Press, United Kingdom, 2020.

[5] A. Blokus, K. Kołowrocki, "Influence of component dependency on system reliability," in: Zamojski W., Mazurkiewicz J., Sugier J., Walkowiak T., Kacprzyk J. (eds.) Theory and Applications of Dependable Computer Systems. DepCoS-RELCOMEX 2020. Advances in Intelligent Systems and Computing, vol. 1173, pp. 105-114. Springer, Cham, 2020.

[6] A. Bondavalli, S. Chiaradonna, F. Di Giandomenico, I. Mura, "Dependability Modeling and Evaluation of Multiple-Phased Systems Using DEEM," IEEE Transactions on Reliability, 53:4, 2004.

[7] L. Chybowski, K. Gawdzińska, "On the Present State-ofthe-Art of a Component Importance Analysis for Complex Technical Systems," Advances in Intelligent Systems and Computing, Volume 445, Springer International Publishing, pp. 691-700, 2016.

[8] J. B. Dugan, "Automated analysis of phased-mission reliability," IEEE Transactions on Reliability, 40:1, 1991.

[9] J. D. Esary, H. Ziehms, "Reliability analysis of phased missions," In: Barlow RE, Fussell JB, Singpurwalla ND, editors. Reliability and fault tree analysis: Theoretical and applied aspects of system reliability and safety assessment. Philadelphia, PA, SIAM, pp. 213-236, 1975.

[10] J. F. Espiritu, D. W. Coit, U. Prakash, "Component criticality importance measures for the power industry," 
Electric Power Systems Research, Vol. 77, Issues 5-6,pp. 407 - 420, 2007.

[11] K. Formela, T. Neumann and A. Weintrit, “Overview of Definitions of Maritime Safety, Safety at Sea, Navigational Safety and Safety in General," TransNav, the International Journal on Marine Navigation and Safety of Sea Transportation, Vol. 13, No. 2, doi:10.12716/1001.13.02.03, 2019, pp. 285-290.

[12] J. Håvold, "The RMS Titanic disaster seen in the light of risk and safety theories and models," In Safety, Reliability and Risk Analysis; CRC Press, pp. 475-482, 2013.

[13] C. Hetherington, R. Flin, K. Mearns, "Safety in Shipping: The human element," Journal of Safety Research, vol. 37, 401 - 411, 2006.

[14] K. Kołowrocki, J. Soszynska-Budny, "Reliability and Safety of Complex Technical Systems and Processes, Modeling - Identification - Prediction - Optimization," Springer-Verlag, 2011.

[15] W. Kuo, X. Zhu, "Importance measures in reliability, risk, and optimization. Principles and application," John Wiley \& Sons, Ltd., 2012.

[16] T. Nowakowski, S. Werbińska, “On problems of multicomponent system maintenance modelling, "Int. J. Autom. Comput. 6, 364, 2009.

[17] Z. Pietrzykowski, "Assessment of Navigational Safety in Vessel Traffic in an Open Area," TransNav, the International Journal on Marine Navigation and Safety of Sea Transportation, Vol. 1, No. 1, pp. 85-88, 2007.
[18] M. Rausand, A. Høyland, "System Reliability Theory: Models, Statistical methods, and Applications (2nd ed.)," Wiley, Hoboken, 2004.

[19] J. Reason, "Human error," Cambrige University Press, New York, 1990.

[20] A.K. Somani, J. A. Ritcey, S. H. L. Au, "Computationally efficient phased-mission reliability analysis for systems with variable configurations," IEEE Transactions on Reliability, 41(4), pp. 504-511, 1992.

[21] M. Stewart, R. Melchers, "Probabilistic Risk Assessment of Engineering Systems," Chapman Hall, London, 1997.

[22] W. Vesely, M. Stematelators, J. Dugan, J. Fragola, J. Minarick, J. Railsback, "Fault Tree. Handbook with Aerospace Applications," NASA Research Center, Washington, 2002.

[23] L. Xing, "Reliability evaluation of phased-mission systems with imperfect fault cover age and commoncause failures," IEEE Transactions on Reliability, 56 (1), pp. 58-68, 2007.

[24] L. Xing, J. B. Dugan, “Analysis of generalized phased mission system reliability, performance and sensitivity," IEEE Transactions Reliability, 51, pp. 199211, 2002.

[25] W. Yueqin and R. Zhanyong, "Mission reliability analysis of multiple-phased systems based on Bayesian network," 2014 Prognostics and System Health Management Conference (PHM-2014 Hunan), Zhangiiaijie, pp. 504-508, 2014, doi: 10.1109/PHM.2014.6988224. 